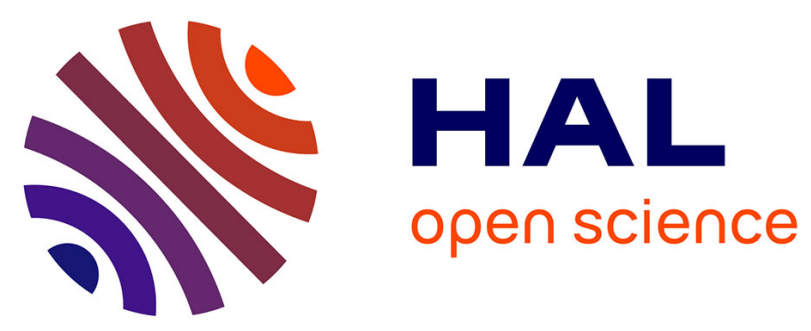

\title{
SURFACE MODIFICATIONS USING EXCIMER LASERS ; FUNDAMENTALS AND APPLICATIONS
}

\author{
H. Bergmann, E. Schubert, K. Schutte
}

\section{To cite this version:}

H. Bergmann, E. Schubert, K. Schutte. SURFACE MODIFICATIONS USING EXCIMER LASERS ; FUNDAMENTALS AND APPLICATIONS. Journal de Physique IV Proceedings, 1991, 01 (C7), pp.C7-7-C7-12. 10.1051/jp4:1991702 . jpa-00250739

\section{HAL Id: jpa-00250739 https://hal.science/jpa-00250739}

Submitted on 1 Jan 1991

HAL is a multi-disciplinary open access archive for the deposit and dissemination of scientific research documents, whether they are published or not. The documents may come from teaching and research institutions in France or abroad, or from public or private research centers.
L'archive ouverte pluridisciplinaire HAL, est destinée au dépôt et à la diffusion de documents scientifiques de niveau recherche, publiés ou non, émanant des établissements d'enseignement et de recherche français ou étrangers, des laboratoires publics ou privés. 


\title{
SURFACE MODIFICATIONS USING EXCIMER LASERS; FUNDAMENTALS AND APPLICATIONS
}

\author{
H.W. BERGMANN, E. SCHUBERT and K. SCHUTTE
}

Forschungsverbund Lasertechnologie Erlangen (FLE), Universität Erlangen-Nürnberg, Lehrstuhl Werkstoffwissenschaft (Metalle), Martensstrasse 5, D-8520 Erlangen, Germany

\begin{abstract}
It is the purpose of the present investigation to describe and to explain the occurring phenomena during the interaction of intensive, pulsed UV-light with technical metal and ceramic surfaces, on the one hand, and based on this knowledge, to show possible technological applications on the other hand.
\end{abstract}

\section{Introduction}

For several years now, laser processing has been an accepted technology commonly applied in industry. The different processes performed with the different types of lasers are shown in the power flux density -interaction time - diagrams, see figure 1. Cutting and welding is possible using cw-lasers with medium power flux densities, whereas new processes and new types of surface modifications can only be achieved using pulsed lasers, e.g. high power excimer lasers. So far, only a few industrial applications of excimer lasers are known, e.g. structuring of microelectronic components /1/ and marking of glasses.

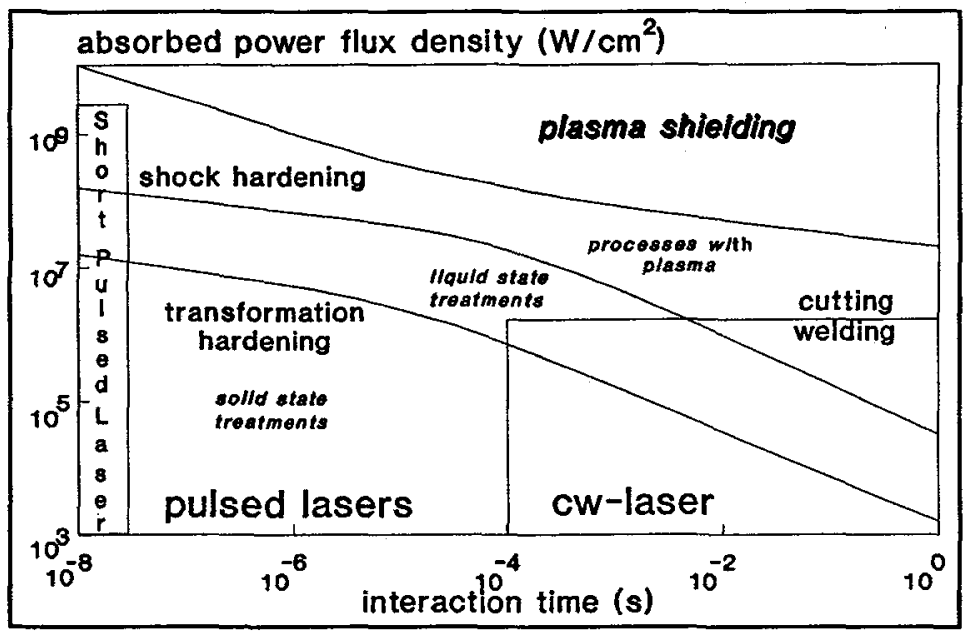

Fig. 1 Power flux density - interaction time - diagram for materials processing with lasers 
2. Experimental investigaticas on the interaction of UV-radiation and technical metal and ceramic surfaces

The fundamental experiments were carried out with a Siemenstype excimer laser (laseractive medium $(\mathrm{XeCl})^{*}$, wavelength $308 \mathrm{~nm}$, pulse duration $40 \mathrm{~ns}$, pulse energy $2 \mathrm{~J}$, repetition rate $20 \mathrm{~Hz}$ ) using the mask projection technique. The evaluation of the results was almost exclusively performed using optical methods such as differential reflectometry $/ 2 /$, because the treatment is always restricted to very thin layers. Parallel to the detection and characterization of the surface changes, the technically interesting properties of the devices (mechanical, electrical, wear or corrosion properties) were tested under service conditions. After studying the influence of the laser parameters and the pre-treatment, the dependance of surface modifications on the physical properties of the different materials was investigated.

\subsection{Influence of the laser parameters}

Using a special type of excimer laser, the wavelength, pulse duration, and maximum repetion rate were determined. Changing the wavelength results in decreased or an increased absorption by the irradiated material. The influence of these parameters can easily be estimated. An increasing pulse duration leads to a higher penetration depth because of the longer interaction time. For surface treatments, the repetition rate is of minor significance. Up to $100 \mathrm{~Hz}$ and energy densities of $40 \mathrm{~mJ} / \mathrm{mm}^{2}$ for metals no thermal influence could be detected. Therefore the power flux density is the most important parameter and normally the only one which can be changed by the user.

The following figure 2 shows the influence of the power flux density on the change of materials properties. The drawing can be applied to different metals and ceramics as the given flux density is normalized over the flux density where the plasma ignition starts (plasma flux density). The different processes occurring with increasing fluxes are demonstrated.

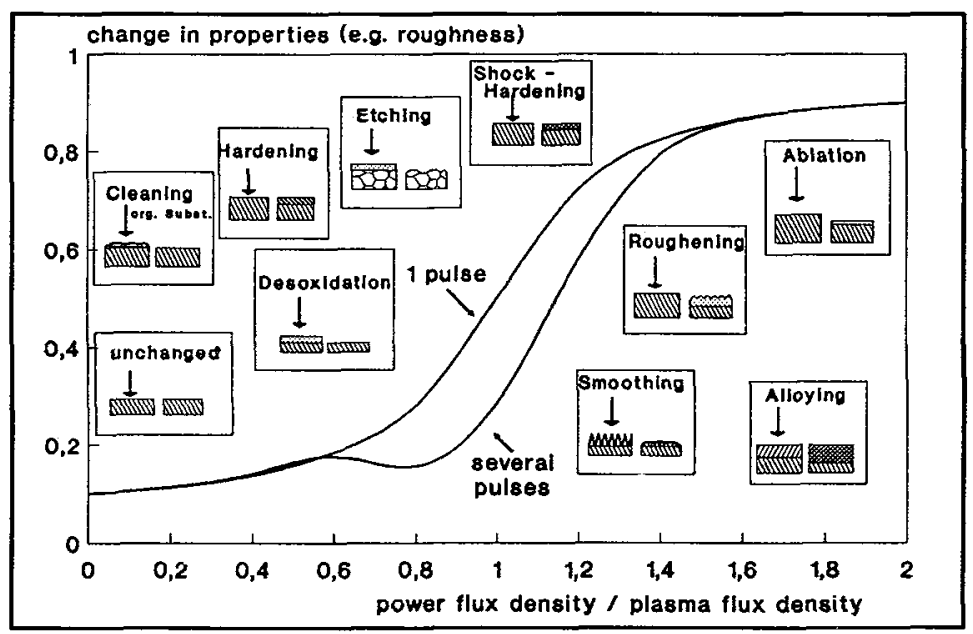

Fig. 2 Possible processes during interaction of intensive UV-light with metal surfaces

\subsection{Influence of the pre-treatment}

Due to the small penetration depth of the laser radiation, the pre-treatment of the substrates is of essential interest. Each production step leaves its finger-prints on the metallic or ceramic samples. A typical technical surface with different layers is shown in figure 3 on top of the next page. 


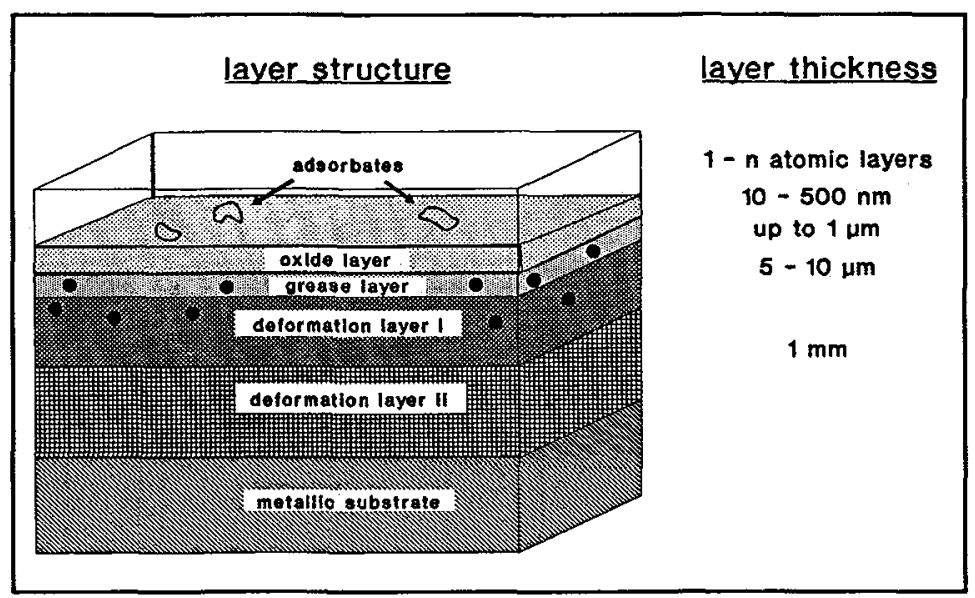

Fig.3 Schematic drawing of a technical metal surface

Depending on the different surface layers, interaction with the laser radiation is changed from a thermal (metallic substrate) to a photolytic (organic or oxyde layers) mechanism. This allows to remove oxyde or deformation layers from the substrate without thermal load.

In addition to the surface modifications generated by the production process, the deviations from ideal single crystal have to be considered. In general, all defects like dislocations, grain boundaries and porosities increase the absorption of the laser radiation. Therefore different parameters are needed to achieve similar surface conditions, e.g. for cold rolled and recrystallized materials.

\subsection{Influence of the material properties}

A systematic investigation was carried out to determine the influence of the materials properties. More then 20 different pure metals and alloys with different crystal structures were examined. Assuming a 1-dimensional heat conduction model, the melting depth can be calculated as a function of the normalized thermal conductivity, see figure 4 .

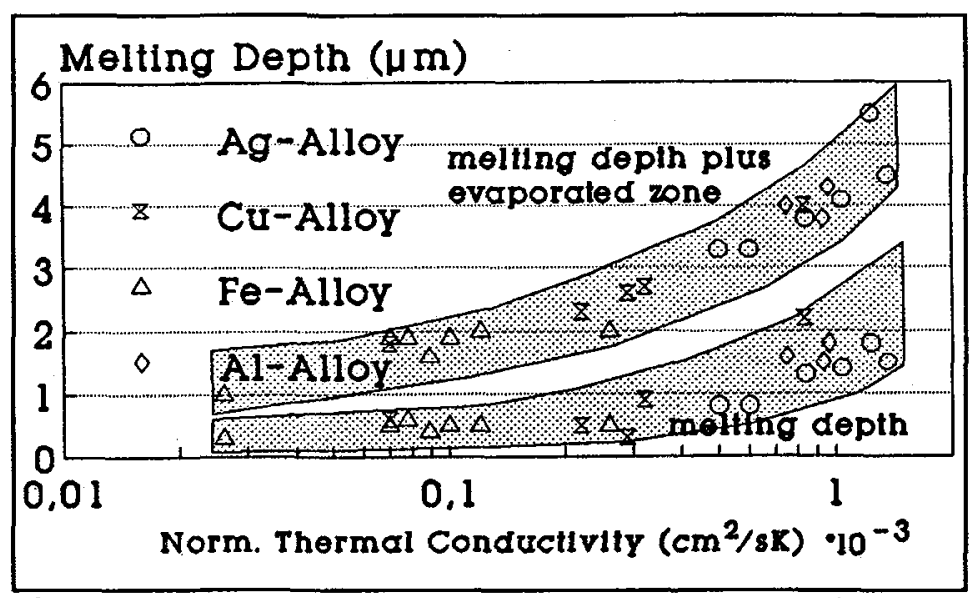

Fig. 4 Melting depth as a function of the physical properties of the alloys investigated 


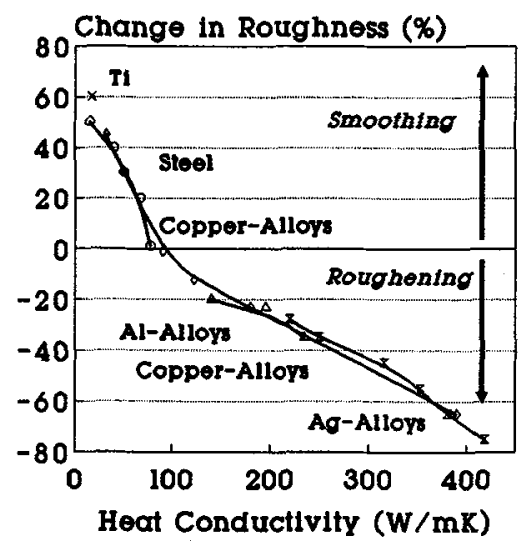

Fig. 5 Surface modification of different alloys as a function of the heat conductivity
The melting depth is mainly influenced by the thermal conductivity of the material. For surface modification, two different regions have to be considered. If the heat conductivity is high, the laser power is transferred into the volume by heat convection very quickly. This results in an increased roughness as the melt does not exist for a time long enough to allow thermal flow processes. For this reason, a smoothing of material surfaces with low heat conductivities can easily be obtained. Figure 5 exhibits the two different regions of smoothing and roughening for the materials investigated.

3. Possible applications for surface treatments of metals and ceramics

The basic know-how gained during the fundamental phase was used to leave the field of the fundamentals of surface modifications and to achieve technological surface treatments. For this purpose, components of different industrial branches (e.g. electronic and automotive industry) were choosen, where surface properties are essential for the function of the devices. Three examples will be discussed more detailed in the following sections.

\subsection{Improving of the bonding behavior of lead frames}

The investigated contact materials were lead frames with copper, nickel, gold, and silver bonding areas.

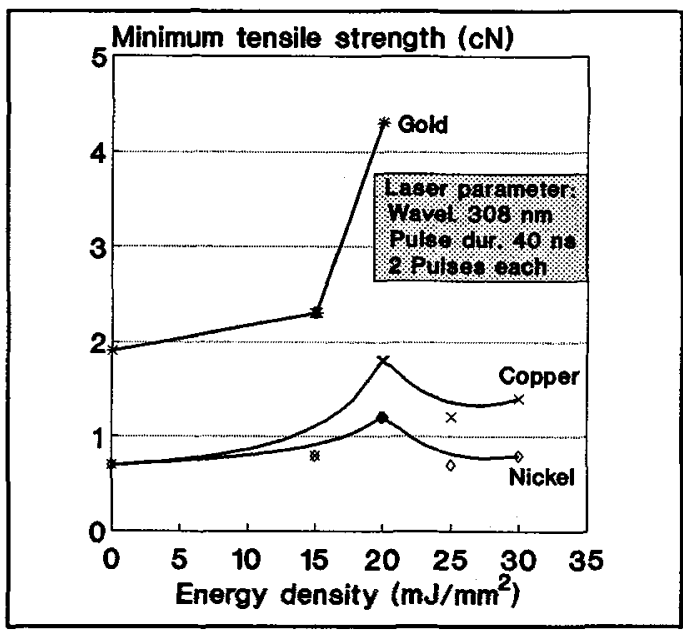

Fig. 6 Bonding behavior of $\mathrm{Au}-, \mathrm{Cu}-$ and $\mathrm{Ni}-$ bonds after excimer laser treatment
The correlation between the decrease of reflectance (measured by means of spectral differential reflectometry), bonding behavior and surface layers generated by annealing the untreated samples to pollution atmosphere could be demonstrated. Figure 6 shows the minimum tensile strength of the different bonds as a function of the applied laser energy density. Up to a value just below the threshold for ignition of a metal plasma on the surface, an improving of the bonding behavior can be achieved. The method of the measurement of the tensile strength with 50 to 500 bond tests gives statistically significant and reproducible values. 


\subsection{Laying bare of graphite for parts of the automotive industry}

The process of optical etching (see fig. 2) by irradiating ferritic-pearlitic nodular cast iron can be an alternative to the industrial processes using a chemical etching technique to avoid pollution problems 13/, 14/.

The laying-bare of the graphite during this process is of great interest, because the excimer laser treatment reopens the graphite inclusions as well and this will lead to an improved self-lubrification in the critical running-in phase of automotive parts. Figure 7 exhibits the influence of the surface roughness on the maximum of free graphite. Because of the small penetration depth of the laser light, there is a small processing window. The original surface roughness should not exceed a minimum roughness threshold for this process. The correlation between reflectance decrease and percentage of opened graphite sheriodals on cast iron samples can be seen in figure 8. With increasing values of openend graphite, the signal amplitude of the reflected light will drop due to higher absorption of the measurement light. This effect can be used for a quality or on-line process control for materials processing with excimer lasers.

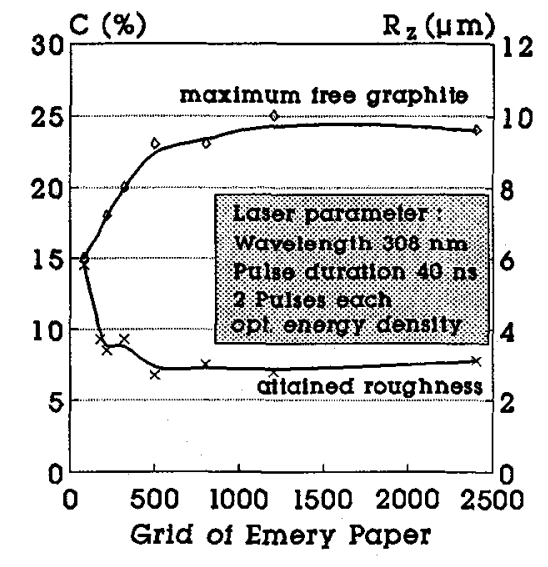

Fig. 7 Laying bare of graphite by excimer laser teatment of nodular cast iron

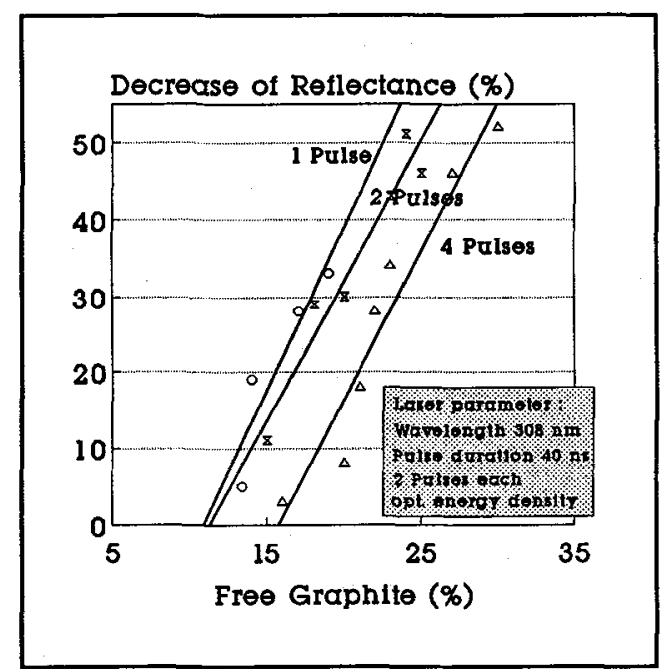

Fig. 8 Correlation between reflectance decrease and percentage of free graphite

\subsection{Improving the wear resistance of alumina samples}

Engineering ceramics are of high interest for electronic and medical applications. The ablation and smoothing of ceramics is a well known process, reported in literature, e.g. 15/. A typical application is the improving of the wear resistance against mechanical and dynamical stresses.

The authors examined the surface treatment of alumina samples using the effect of sealing the ceramic surface by simultaneously smoothing and reduction of the surface porosity. The influence of the pretreatment and the purity on the bending strength and Weibull modulus is detected $/ 6 /$. In figure 9 the improving of the weibull-modulus as a function of the energy density of the laser irradiation and the influence of the purity can be seen. The Weibull modulus could be increased twice compared to the untreated samples. Therefore, the laser treated samples will have a much lower percentage of failure in an industrial process. 


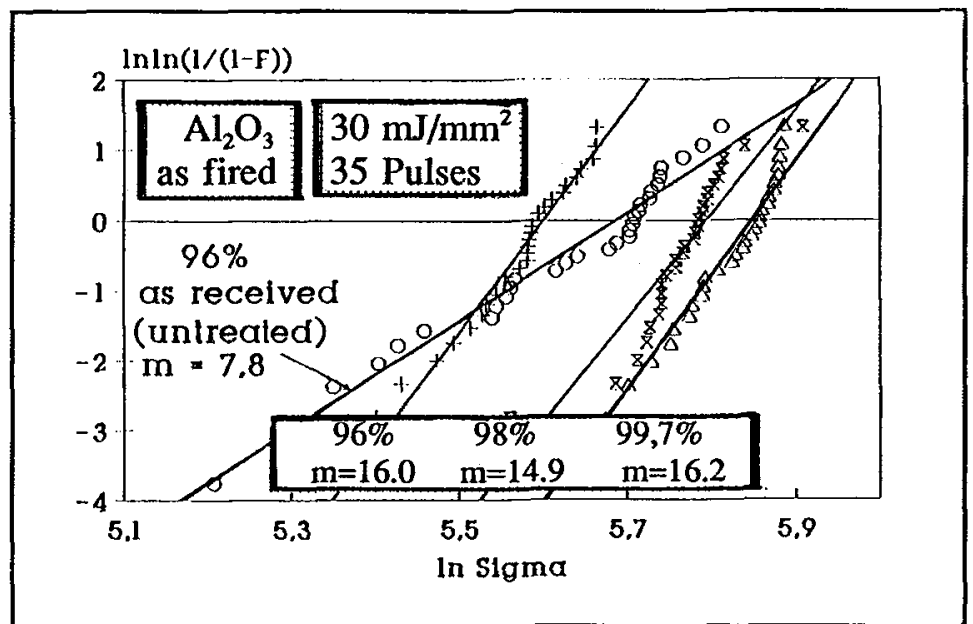

Fig. 9 Weibull modulus as a function of pre-treatment and purity for alumina samples

\section{Conclusions}

Excimer lasers with uniform beam profile and high pulse energy offer interesting possibilities for materials processing of metals and ceramics. Due to the wavelength and penetration depth, the excimer laser is an excellent working tool for surface treatments without damaging the workpieces. If the critical problems for industrial application (e.g. beam homogenizing, beam shaping and guiding, reliability of electrical circuit components and an on-line process control) can be solved in the future, a wide range of application for these new processes can certainly be anticipated.

\section{References}

/1/ F. Bachmann

Application of excimer lasers in an industrial fabrication line

Proc. of the ECLAT'90, Sprechsaal Publishing Group, Coburg (1990), Vol. 2, p. 825

12/ S.M. Rosiwal

Surface treatments with the excimer laser and quality control by means of difference reflectometry

Proc. of the ECLAT‘90, Sprechsaal Publishing Group, Coburg (1990), Vol. 2, p. 895

13/ G. Barton, H.W. Bergmann, E. Schubert, M. Wehner, K. Schmidt

Application of excimer lasers in car body production

Proc. of the 2. Int. Application Forum, BIAS, Bremen (1990), p. 142

14/ E. Schubert, H.W. Bergmann

Finishing techniques for metals and ceramics using excimer lasers

Proc. of the ECLAT'90, Sprechsaal Publishing Group, Coburg (1990), Vol. 2, p. 905

15/ H.K. Tönshoff, O. Gedrat

Removal process of ceramic materials with excimer laser radiation.

Proc. SPIE, Vol. 1132 (1989), p. 104

/6/ E. Schubert, H.W. Bergmann, M. Hald

Modification of ceramic materials using excimer lasers

Proc. of the 10th Int. Congress LASER'91, Springer Verlag, Berlin (1991), in print 